\title{
DESENVOLVIMENTO DE MÉTODO PARA ESPECIFICAÇÃO DE SOQUETES E EXTENSÕES DURANTE ANÁLISE VIRTUAL DA MONTAGEM DOS VEÍCULOS NA INDÚSTRIA AUTOMOTIVA
}

Nivea Brito da Silva ${ }^{1}$

1 SENAI CIMATEC; Salvador/Bahia; niveabrito@gmail.com

Resumo: Esse trabalho apresenta o desenvolvimento e resultado da aplicação de um método para auxiliar, durante a fase de montagem virtual do veículo, o processo de especificação e compra de soquetes e extensões a serem anexados em máquinas rotativas de aperto (apertadeiras) utilizadas na linha de produção automotiva. Para isso, passos metodológicos foram seguidos, iniciando por um diagnóstico com alguns profissionais que atuam na atividade com o objetivo de analisar seus principais desafios. Como resultado, foi constituído um banco de dados em formato de Excel, o qual reune e traduz as informações disponibilizadas pelos fabricantes dos soquetes e extensões em seus catálogos técnicos. Por fim, o estudo demonstra que o resultado obtido foi satisfatório.

Palavras-Chave: Manufatura automotiva avançada; Power tool; Apertadeira; Soquete; Extensão.

\section{DEVELOPMENT OF METHOD TO SPECIFICATION OF SOCKETS AND EXTENSIONS DURING VEHICLE VIRTUAL ANALYSIS IN AUTOMOBILISTIC INDUSTRY}

\begin{abstract}
This article shows a methodology developed and applied to helps, during virtual vehicle assembly phase, the process of specification and purchase of sockets and extensions that needs to be attached to electric power tools used on line production assembly parts to vehicle, describing some benefits that maintenance team got with this implementation.
\end{abstract}

Keywords: Automotive Manufacturing; Power tool; Socket; Extension. 


\section{INTRODUÇÃO}

Diante de um mercado cada dia mais competitivo, aliado com a situação de crise política e econômica que o Brasil vem enfrentando nos últimos anos, o conceito de fazer mais com menos nunca foi tão difundido e aplicado, seja na esfera dos lares, na indústria ou comércio em geral. Tratando-se de indústria, as companhias têm interesse em economizar recursos desde o copo plástico do cafezinho até equipamentos caros e robustos.

O conceito da palavra manutenção é trazido pela Norma Brasileira 5462 Confiabilidade e Mantenabilidade (NBR, 1994) como "combinação de todas as ações técnicas e administrativas, incluindo as de supervisão, destinadas a manter ou recolocar um item em um estado no qual possa desempenhar uma função requerida" está fortemente relacionada a economia de recursos, que se traduz em economia de dinheiro. Quanto mais é mantido o que se tem, menos se gasta em novas aquisições e consequentemente mais dinheiro é poupado (dadas exceções onde a troca é mais vantajosa e caberá uma análise de ciclo de vida - LCCA), além dos benefícios ambientais, que são incomensuráveis.

Conforme descrevem [1], "é importante considerar a padronização com outros equipamentos do mesmo projeto e com equipamentos já existentes na instalação, objetivando redução de estoque de sobressalentes e facilidades na manutenção e operação". Este trecho ressalta a importância de tópicos que são adjacentes ao ato de consertar e influencia diretamente no bom desempenho e funcionamento de todo sistema de manutenção. Quanto mais minuciosa for a fase de projeto dos equipamentos e/ou sistemas em geral, menor será a quantidade de problemas encontrados após o período transitório de utilização.

Falando especificamente da montagem automotiva, quanto mais perfeito for 0 desenho de uma determinada peça e do seu processo de implementação na linha de produção, menores serão os imprevistos durante essa implementação e consequentemente a quantidade de vezes que o time da manutenção será acionado para cobrir eventuais falhas também reduzirá. Por isso, melhorando a confiabilidade nas fases iniciais do projeto, a reação em cadeia será diminuir a atuação do time de manutenção na linha de produção.

\subsection{Justificativa}

O trabalho se justifica devido a existência de lacunas quanto a registro de material orientativo, seja ele em versão física ou digital, que auxilie os profissionais na definição do código comercial de compra (part number) de soquetes e extensões. Esse tipo de trabalho é realizado esporadicamente, a medida que novos grandes projetos são aprovados e executados. E a baixa frequência neste tipo de atividade contribui para que os profissionais que atuam no processo esqueçam como fazê-lo.

\subsection{Objetivo}


O objetivo do trabalho é desenvolver e aplicar uma ferramenta, utilizando como referência o banco de dados extraído dos catálogos de fabricantes, que auxilie na definição do part number de soquetes e extensões a partir de itens virtuais e especificações do processo de montagem, utilizados em simulações virtuais 3D de montagem dos veículos.

\section{METODOLOGIA}

Para elaboração da pesquisa foram realizadas as seguintes etapas: (I) busca por material orientativo já existente. Verificando bancos de dados tanto na sede da companhia onde o estudo foi realizado, quanto de outros países, foi notada a falta de um guia que auxilie a escolha e compra de soquetes e proteções utilizadas na montagem automotiva. Após este primeiro levantamento de dados, o trabalho seguiu as demais etapas: (ii) entrevista com funcionários para entender suas dificuldades com o a interpretação dos catálogos técnicos dos fabricantes, através de formulário diagnóstico; (iii) consulta a esses catálogos; (iv) construção do banco de dados no Excel; ( $v$ ) realização de testes e verificação de feedback dos usuários e; (vi) divulgação e implantação da ferramenta para todo o departamento da planta que trabalha ou tem potencial de desenvolver essas atividades.

Figura 1. Exemplo de simulação virtual 3D para verificar acesso de ferramenta de aperto

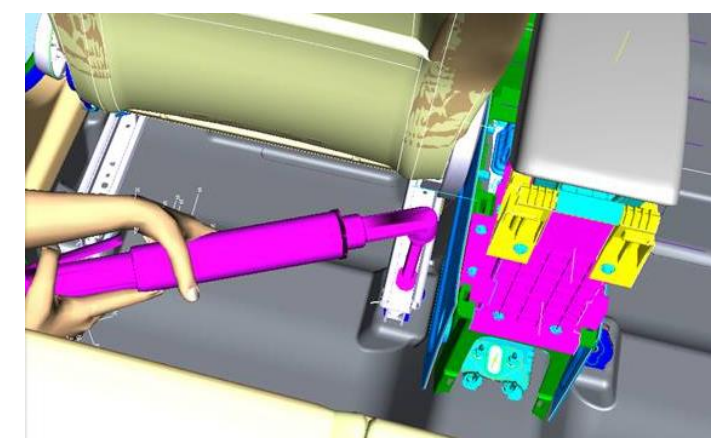

Fonte: Elaborado pela autora através do software Teamcenter (2019).

O catálogo do fabricante das soquetes e extensões possui informações sobre centenas de produtos que ele tem a oferecer. A marca utilizada nesse estudo de caso foi selecionada devido ser uma das homologadas (e a mais utilizada) pela companhia, que possui rigorosos critérios de qualidade e resistência para soquetes de alto impacto. Vale ressaltar que, durante a montagem final do veículo são utilizados poucos tipos de itens desse catálogo, porém se repetindo dezenas de vezes. Isso ocorre pois a maioria das operações é padrão, ainda que novos carros sejam lançados. Como pode ser visto nas Figuras $3 a$ e $3 b$, além da métrica, para todos os itens, existe a opção de detalhamento pela unidade de medida padrão SAE (Society of Automotive Engineers - EUA) que é uma classificação padronizada mundialmente por esse órgão. 
Figura 3a. Unidade de medida SAE

\section{3/8" Square Drive Sockets, SAE}

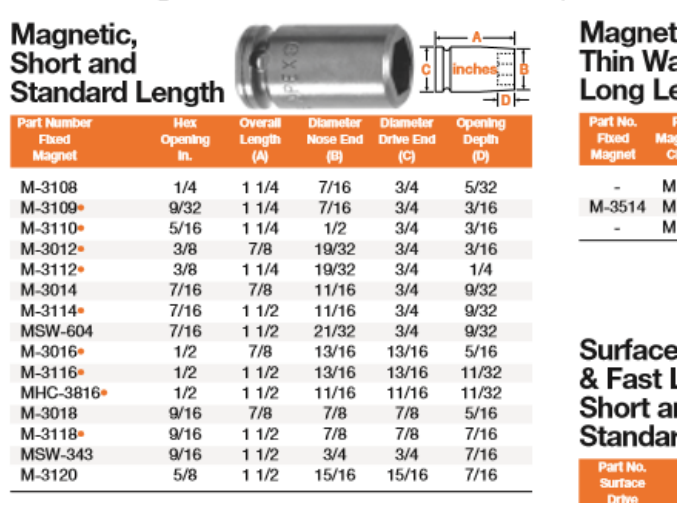

Figura 3b. Unidade de medida métrica

\begin{tabular}{|c|c|c|c|c|c|}
\hline 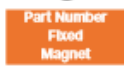 & $\begin{array}{l}\text { Hex } \\
\text { openng } \\
m m\end{array}$ & $\begin{array}{l}\text { Ovaras } \\
\text { Longth } \\
\text { (A) }\end{array}$ & $\begin{array}{l}\text { Dianeara } \\
\text { Nose End } \\
\text { (B) }\end{array}$ & $\begin{array}{l}\text { Diangater } \\
\text { Dine End } \\
\text { (C) }\end{array}$ & $\begin{array}{l}\text { openthy } \\
\text { Deph } \\
\text { (i) }\end{array}$ \\
\hline M- $6 \mathrm{~mm} 13$ & 6 & 31.8 & 11.1 & 19.1 & 3.9 \\
\hline M-7mm13. & 7 & 31.8 & 11.1 & 19.1 & 4.8 \\
\hline M-8mm13. & 8 & 31.8 & 12.7 & 19.1 & 4.8 \\
\hline M-9mm13 & 9 & 31.8 & 15.1 & 19.1 & 4.8 \\
\hline M-10mm03. & 10 & 22.2 & 16.8 & 19.1 & 6.4 \\
\hline M-10mm13. & 10 & 31.8 & 16.8 & 19.1 & 6.4 \\
\hline M-11mm03 & 11 & 22.2 & 17.5 & 19.1 & 7.2 \\
\hline M-11mm13. & 11 & 38.1 & 17.5 & 19.1 & 7.2 \\
\hline $\mathrm{M}-12 \mathrm{mm03}$ & 12 & 22.2 & 19.1 & 19.1 & 7.2 \\
\hline M-12mm13. & 12 & 38.1 & 19.1 & 19.1 & 7.2 \\
\hline $\mathrm{M}-13 \mathrm{~mm} 03$. & 13 & 22.2 & 20.6 & 20.6 & 8.7 \\
\hline M-13mm13. & 13 & 38.1 & 20.6 & 20.6 & 8.7 \\
\hline M-14mm13. & 14 & 38.1 & 22.2 & 22.2 & 11.1 \\
\hline M-15mm03. & 15 & 23.8 & 22.2 & 22.2 & 9.5 \\
\hline M-15mm13. & 15 & 38.1 & 22.2 & 22.2 & 11.1 \\
\hline M-16mm13. & 16 & 38.1 & 23.8 & 23.8 & 11.1 \\
\hline M-17mm13. & 17 & 38.1 & 25.4 & 25.4 & 11.1 \\
\hline M-18mm03. & 18 & 25.4 & 25.4 & 25.4 & 11.1 \\
\hline M-18mm13. & 18 & 38.1 & 25.4 & 25.4 & 11.1 \\
\hline
\end{tabular}

Fonte: [2] Catálogo do fabricante (2006).

Os soquetes e extensões que encaixam em máquinas da família ST (elétricas, transdutorizadas - fornecem controle e rastreabilidade de torque, com cabeça tipo macho e quadrado geralmente $3 / 8$ " ou $1 / 2$ ", não servem para máquinas da familia DL (elétricas, não-transdutorizadas, com cabeça tipo macho e quadrado geralmente de 1/4") nem BCP (Battery Control Powertool - à bateria, não-transdutorizadas, com cabeça tipo fêmea e encaixe de forma específica). Na Figura 4 podem ser vistos, respectivamente, três modelos de máquinas elétricas rotativas (apertadeiras), sendo a primeira BCP, a segunda da família ST e a terceira da família DL.

Figura 4. Exemplos de máquinas (apertadeiras) rotativas
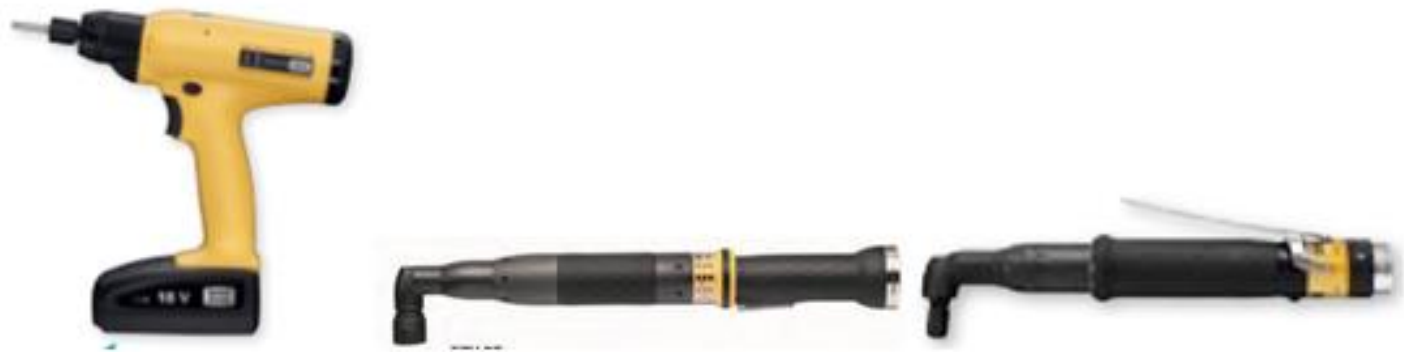

Fonte: [3] Catálogo do fabricante (2016)

Já nas Figuras 5a e 5b são mostrados exemplos de soquetes e extensões, sendo na $5 a$ onde aparecem avulsos e $5 \mathrm{~b}$ em utilização, anexados numa máquina.

Figura 5a. Extensão e soquete, respectivamente 


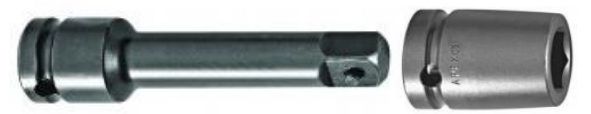

Fonte: [2] Catálogo do fabricante (2006)

Figura 5b. Exemplo de operação com conjunto apertadeira, extensão e soquete

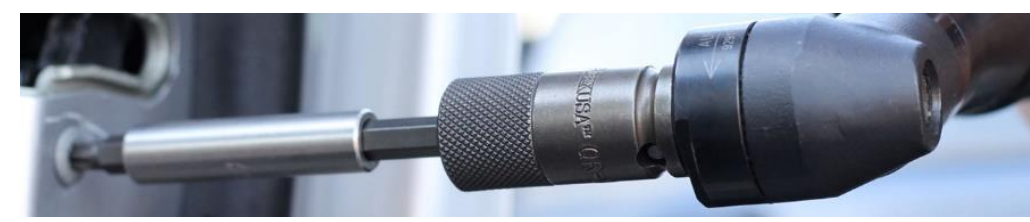

Fonte: [3] Catálogo do fabricante (2016)

O estudo foi realizado numa empresa do setor automotivo, localizada no estado da Bahia, no período entre Abril a Dezembro de 2018. Foram entrevistadas 5 pessoas no total, todas envolvidas na execução dessas atividades. Após o levantamento dos dados, as entrevistas com os funcionários e consulta a catálogos técnicos iniciou-se o desenvolvimento da ferramenta para auxiliar o processo de especificação e compra de soquetes e extensões durante a fase de montagem virtual do veículo.

A ferramenta desenvolvida como auxílio trata-se de um arquivo em Excel, com abas separadas para cada um dos modelos de soquetes a serem especificados da seguinte maneira:

1) A primeira aba mostra a lista de todas as opções de soquetes hexagonais utilizados em máquinas das famílias ST e DL;

2) A segunda aba traz a lista de todas as opções de extensões utilizados em máquinas das famílias ST e DL;

3) A terceira aba mostra a lista de todas as opções de bits torx (ponta de uma ferramenta cujo formato de encaixe do parafuso é caracterizado por uma chave de 6 pontas, formando uma estrela) utilizados em máquinas da família BCP;

4) A quarta aba traz a lista de todas as opções de bit holders (adaptadores para máquinas com cabeça "fêmea", a exemplo das BCPs, que sustentam os bits) e adaptadores (suportes para bits e soquetes, listados na aba 3) que permitem a conexão entre as máquinas da família BCP e os bits e soquetes das abas 1 e 3.

As definições devem ocorrer a partir da primeira coluna à esquerda, de acordo com as especificações desejadas. A medida que os itens forem selecionados nas colunas seguintes, o intervalo de códigos para os soquetes diminui, até que se chegue ao objetivo daquele que atenderá a operação, um part number específico a ser adicionado na solicitação de compra. Caso o operador da planilha deseje observar informações adicionais do item, o mesmo pode ser localizado (através de ferramente de busca) no catálogo do fabricante. A Tabela 1 ilustra parte da primeira aba da planilha em questão. Sem essa ferramenta, originalmente é necessário abrir o catálogo do fornecedor e observar com muito cuidado, dento de milhares de 
informações e códigos não aplicados a processos automotivos, qual é o código correto de acordo com as premissas de projeto conhecidas até então.

Tabela 1. Parte da planilha desenvolvida

\begin{tabular}{|l|c|c|c|c|c|c|c|}
\hline Máquina & $\begin{array}{c}\text { Cabeça } \\
\text { da } \\
\text { Máquina }\end{array}$ & $\begin{array}{c}\text { Tipo de } \\
\text { Fixação }\end{array}$ & $\begin{array}{c}\text { Cabeça do } \\
\text { parafuso/porca }\end{array}$ & Imã? & $\begin{array}{c}\text { Diâmetro } \\
\text { da } \\
\text { cabeça }\end{array}$ & $\begin{array}{c}\text { Comprimento } \\
\text { do soquete }\end{array}$ & $\begin{array}{c}\text { Part } \\
\text { Number }\end{array}$ \\
\hline DL & $1 / 4 "$ & $\begin{array}{r}\text { Parafuso/Porca } \\
\text { (Stud) }\end{array}$ & Hexagonal & $\mathrm{N}$ & 3.5 & Normal & $3.5 \mathrm{~mm} 11$ \\
\hline DL & $1 / 4 "$ & $\begin{array}{r}\text { Parafuso/Porca } \\
\text { (Stud) }\end{array}$ & Hexagonal & $\mathrm{N}$ & 4 & Normal & $4 \mathrm{~mm} 11$ \\
\hline DL & $1 / 4 "$ & $\begin{array}{r}\text { Parafuso/Porca } \\
\text { (Stud) }\end{array}$ & Hexagonal & $\mathrm{N}$ & 4.5 & Normal & $5 \mathrm{~mm} 11$ \\
\hline
\end{tabular}

Fonte: Elaborado pela autora (2019)

\section{CONCLUSÃO}

As atividades industriais precisam ser sistematizadas e registradas. É fundamental para a sustentabilidade dos negócios que o máximo de informações possíveis relacionadas ao processo estejam inventariadas e disponíveis aos funcionários, evitando retrabalhos e economizando recursos.

Não só o corpo de engenharia quanto o "chão de fábrica" devem ser estimulados a fazerem esses registros, sistematizando 0 uso dos recursos/ferramentas e principalmente, utilizá-los o máximo possível. Neste aspecto, idéias aparentemente simples podem se tornar valiosas ferramentas de consulta. Essa pesquisa contribui para que novos funcionários da companhia consigam desenvolver o trabalho sem necessitar da ajuda majoritária dos colegas antigos que já possuem o conhecimento e esses, por sua vez, conseguirá desenvolver o trabalho que já estão acostumados num intervalo de tempo menor, comprando itens corretos da primeira vez. Assim, a companhia ganha na redução de custos e recursos, eliminando retrabalhos, e, sobretudo, garantindo a melhoria da qualidade dos serviços. Pode-se concluir que a elaboração da ferramenta apresentada neste trabalho detalha o passo-a-passo explicitado e sistematizado, dando visibilidade a um conhecimento até então estritamente tácito (o tipo de conhecimento que está apenas na cabeça das pessoas, sem registro físico oficial) e passa a ser um documento de instrução disponível para todos os funcionários da companhia.

\section{Agradecimentos}


Agradeço a minha família por todo amor incondicional. A Mari, orientadora desse trabalho, pelo suporte e incentivo.

\section{REFERÊNCIAS}

${ }^{1}$ KARDEC, Alan; NASCIF, Júlio. Manutenção: Função Estratégica. 3. ed. rev.atual. Rio de Janeiro: QUALITYMARK EDITORA, 2009.

2 APEX®. Disponível em https://www.apex-tools.com/sites/www.apextools.com/files/Apex-Fastening-Tools TC-100-EN.pdf. Acesso em 15/09/2018.

3 Atlas Copco®. Catálogos. Disponível em: https://www.atlascopco.com/pt$\mathrm{br} /$ itba/about-industrialtechnique/brochures-and-catalogs/brochures. Acesso em 15/09/2018.

${ }^{4}$ AGUIAR, Silvio. Integração das Ferramentas de Qualidade ao PDCA e ao Programa Seis Sigma / Silvio Aguiar. Nova Lima: INDG - Tecnologia e Serviços Ltda., 2006. 